numbers of CMs for therapeutic cell transplantation. This study investigated the effect of co-culturing with native CMs on ascorbic acid-induced cardiomyogenic differentiation in embryonic stem cells, to develop a novel protocol for generating functional CMs from ESCs.

Methods Native CMs were isolated from the hearts of 1-day-old Sprague-Dawley rats. Mouse ESCs were cultured in hanging drops to form embryoid bodies (EBs) and treated with or without $0.1 \mathrm{mM}$ of ascorbic acid (Sigma) for cardiomyogenic differentiation. They were divided into four groups: ascorbic acid \& co-culture group, co-culture group, ascorbic acid group, and control group. In the coculture system, EBs were co-cultured with native CMs by the hanging cell culture inserts (PET $1 \mu \mathrm{m}$ ) (Millicell; Millipore, Bedford, MA, USA). The native CMs were purposely placed on culture plate inserts to prevent direct contact with subnatant EBs. Both the ESCs and native CMs grew in the same medium but they were easy to separate. The structural and functional properties of ESC-derived CMs (ESCM) were evaluated by microscopic observation, immunocytochemistry, RT-PCR, and transmission electron microscopy.

Results The average percentages of EBs exhibited rhythmic contractions in co-culture and ascorbic acid group, co-culture group, ascorbic acid group, and control group were $86.6 \pm 9.52 \%, 65.60 \pm 10.77 \%$, $29.6 \pm 6.03 \%$, and $17.76 \pm 5.99 \%$, respectively. The percentage of beating EBs in co-culture \& ascorbic acid group was much higher and the homogeneity of EBs were significantly improved over that seen in other groups $(p<0.01)$, simultaneously, the automaticity of beating also maintained for more time. The majority (>90\%) of cells in EBs were ESCM that acquired almost the same structural and functional properties as typical CMs.

Conclusions The present study demonstrates the cardiomyogenic differentiation of ESCs can be efficiently controlled by co-cultured with native CMs, and this may lead to a practicable cocktail approach to generate ESC-derived CMs for stem cell-based regenerative medicine.

\section{e0231 POLYMER-FREE SIROLIMUS AND PROBUCOL-ELUTING STENT FOR RENAL ARTERY: AN INITIAL EXPERIENCE IN SWINE}

doi:10.1136/hrt.2010.208967.231

Zheng Bin, Yan Hongbing. Beijing Anzhen Hospital, Beijing, China

Objective Bare metal stent (BMS) implantation can resolve renal artery stenosis successfully, but in-stent restenosis does occur, especially in small diameter renal arteries $(<5.0 \mathrm{~mm})$. The aim of this study is to test whether a newly designed polymer-free sirolimus and probucol-eluting stent (SPES) can inhibit neointimal hyperplasia of renal artery in swine.

Methods 26 stents (18 SPES and 18 BMS) were implanted in 36 renal arteries of 18 animals. During every procedure, a SPES and a BMS were randomised to the right or left renal artery. Seven animals were sacrificed after 90 days, and 11 after 180 days.

Results Histomorphometric analysis was performed. After 90 days, minimal lumen area, neointimal area, score of inflammation and score of endothelialisation were not significantly different between BMS and SPES. After 180 days, minimal lumen area was not significantly different between BMS and SPES $\left(6.55 \pm 2.91 \mathrm{~mm}^{2}\right.$ vs $\left.7.32 \pm 1.99 \mathrm{~mm}^{2}, \mathrm{p}=0.477\right)$, but neointimal area was significantly less in SPES vessels than in BMS vessels $\left(3.07 \pm 0.83 \mathrm{~mm}^{2}\right.$ vs $\left.4.47 \pm 1.23 \mathrm{~mm}^{2}, p=0.005\right)$. Score of inflammation and score of endothelialisation were not significantly different between BMS and SPES after 180 days.

Conclusion SPES can successfully inhibit neointimal hyperplasia of renal artery in swine. At the same time, inflammation and endothelialisation in SPES vessels were similar to BMS vessels.

\section{e0232 THE CHANGE OF VENTRICULAR INA AT DIFFERENT TIME OF SIMULATED ISCHAEMIA AND THE EFFECT OF ATORVASTATIN}

doi:10.1136/hrt.2010.208967.232

Li Hongshi, Bian Bo, Wan Zheng, Teng Tianming, Wang Fang. Tianjin Medical University General Hospital, Tianjin, China

Objective To observe time dependent effects of simulated ischaemia on transient sodium currents $\left(\mathrm{I}_{\mathrm{Na}}\right)$ of rat left ventricular myocytes, and the effects of atorvastatin on ischaemia $\mathrm{I}_{\mathrm{Na}}$.

Methods 30 Wistar rats were used for isolating left ventricular myocytes, which were randomly divided into two groups: ischaemia group (normal $\rightarrow$ simulated ischaemia) and statin group (normal $\rightarrow$ simulated ischaemia with $5 \mu \mathrm{mol} / 1$ atorvastatin). $\mathrm{I}_{\mathrm{Na}}$ were recorded in normal condition (for control) by whole-cell patchclamp. Then in simulated ischaemia condition, $\mathrm{I}_{\mathrm{Na}}$ were recorded from 3 to $21 \mathrm{~min}$, monitored normalised peak currents every $2 \mathrm{~min}$, and compared gate parameters between normal and simulated ischaemia ( $3 \mathrm{~min}$ ) condition.

Results a. Normalised currents (at $-40 \mathrm{mV}$ ), in ischaemia group, compared with normal $(0.95 \pm 0.04)$, the currents in simulated ischaemia were increased to peak at $3 \mathrm{~min}(1.15 \pm 0.08, \mathrm{p} 0.05$, respectively), and decreased at $21 \mathrm{~min}(0.56 \pm 0.13, \mathrm{p} 0.05)$. b. Gate parameters, from normal to simulated ischaemia condition at $3 \mathrm{~min}$, membrane potential at $50 \%$ maximal activation $\left(\mathrm{V}_{1 / 2, \mathrm{a}}\right)$, offsetting of activation curve $\left(\mathrm{K}_{\mathrm{a}}\right)$, membrane potential at $50 \%$ maximal inactivation $\left(\mathrm{V}_{1 / 2, \mathrm{i}}\right)$ and deinactivation constant $(\tau)$ were decreased $(\mathrm{p}<0.01$, respectively) in ischaemia group, but offsetting of inactivation curve $\left(\mathrm{K}_{\mathrm{i}}\right)$ were not changed; compared between two groups, $K_{i}$ of statin group were decreased $(p<0.05)$ and the decrease of $\tau$ value in statin group were less than ischaemia group $(p<0.05)$.

Conclusions The effects of simulated ischaemia on $\mathrm{I}_{\mathrm{Na}}$ are time dependent, while $\mathrm{I}_{\mathrm{Na}}$ is transient increased at $3 \mathrm{~min}$, and atorvastatin can depress this process.

\section{e0233 LATE GADOLINIUM-ENHANCED MRI IN RESTRICTIVE CARDIOMYOPATHY}

doi:10.1136/hrt.2010.208967.233

Zhao Shihua, Jiang Shiliang, Cheng Huaibing, Lu Minjie, Ling Jian, Zhan Yan, Yan Chaowu, Liu Oiong, Ma Ning, Li Shiguo, Yin Gang. Department of Radiology, Cardiovascular Institute and Fuwai Hospital, Chinese Academy of Medical Sciences, Peking Union Medical College, Beijing, China

Objective To evaluate the diagnostic value of MRI in combination of late gadolinium-enhanced imaging in the identification of restrictive cardiomyopathy (RCM).

Methods 116 patients with RCM underwent ECG, x-ray, Echocardiography and MRI. The final diagnosis was made on comprehensive evaluation in consideration of patients' history, clinical symptom and sign, imaging modalities. All patients had objective evidence of impaired cardiac filling and were referred to rule out pericardial thickening. Five histologically proven cases with RCM included heart transplantation in four patients with RCM, endomyocardial biopsy in one patient with RCM. Fifty-five normal subjects were used for reference. All patients were divided into two groups according to contrast-enhanced MRI: RCM with delayed enhancement (RCM with DE, $n=35$ ) and RCM without delayed enhancement (RCM without DE, $n=81$ ). Quantitative measurement of bi-atrial and bi-ventricular size, ventricular septal and left free wall thickness were done. A paired t-test was used for statistic analysis and a $\mathrm{p}$ value of less than 0.05 was considered significant. Qualitative assessment of segmental wall motion, in this present study.

Results The parameters, such as bi-atrial size, right ventricular diastolic diameter (RVDD), ventricular septal and left free wall 